\title{
REFRACTION IN CHILDREN WITH UNILATERAL AND BILATERAL CONGENITAL NASOLACRIMAL DUCT OBSTRUCTION
}

\author{
Krasina P. Valcheva, \\ Snejana V. Murgova
}

Division of Ophthalmology, Medical University - Pleven, Bulgaria

\section{Corresponding Author:}

Krasina P. Valcheva

Division of Ophthalmology, Medical University - Pleven 91, Gen.Vl.Vazov Str.

Pleven, 5800

Bulgaria

e-mail: krasina_valcheva@abv.bg

Received: January 15, 2019

Revision received: March 06, 2019

Accepted: July 30, 2019

\section{Summary}

The study aimed to identify the type of refraction in children with unilateral and bilateral congenital nasolacrimal duct obstruction (CNLDO) who underwent nasolacrimal duct probing. One hundred and twelve children aged 1.8 to 13 years, diagnosed with CNLDO, were included in a retrospective study. All the patients had their visual acuity examined, and their refractive status was evaluated. The refractive errors of both eyes were noted, followed by an appropriate management plan. Of the 112 children, 79 (70.5\%) had a unilateral obstruction, and 33 (29.5\%) had bilateral obstruction. Nine children (11.4\%) in the former group and six children (18.2\%) in the latter group were with visual acuity $<0.8$. The most frequent refractive error among the studied children was hyperopia. No statistically significant difference $(p>0.05)$ between refraction of the two eyes in the evaluated children were found. Amblyopia risk factors were identified in 9 of the patients $(8.0 \%)$. CNLDO did not adversely affect the refractive status. However, amblyogenic risk factors were found more often in our patients, as compared to general populations. Therefore, a detailed evaluation should be performed to find out these risk factors.

Key words: refractive status, risk factors, congenital nasolacrimal duct obstruction

\section{Introduction}

Congenital nasolacrimal duct obstruction (CNLDO) is the most common abnormality of the lacrimal drainage system in ophthalmological practice, affecting $70 \%$ of newborns [1].

The condition is usually due to failure of canalization of the nasolacrimal duct (NLD) at its distal end [2] and causes tearing and secretion in babies eyes [3]. CNLDO has been recognized as a benign disease, with no association with the development of the eye. In the last twenty years, there are works reporting information on related refractive anomalies and risk factors for amblyopia development in children with CNLDO. The preponderance of risk factors varies between $9.5 \%$ and $22.0 \%[4,5]$. The most frequent refractive error among children with CNLDO is hyperopia [6]. Some authors have found that children with this problem manifest 
anisometropia or amblyopia more often than children in the general population [7]. Other authors have concluded that affected subjects have a higher percentage of amblyopia in the eye with a history of CNLDO [8]. Laterality is also important. Unilateral CNLDO may be a risk factor for anisohypermetropia [6]. Cases with bilateral CNLDO are at a much lower risk of developing significant anisometropia and anisometropic amblyopia [9].

The purpose of this study was to assess the refraction in children with unilateral and bilateral CNLDO who underwent NLD probing.

\section{Patients and Methods}

A retrospective study was undertaken at the Eye Clinic in the University Hospital-Pleven, Bulgaria. The Commission of the Ethics of Research Activities in the Medical University of Pleven reviewed and approved the protocol for this study.

Examination of the medical records for a period of 10 years (from 2006 to 2016) identified 224 children with CNLDO who had undergone probing and irrigation of the nasolacrimal duct for tearing and discharge since early infancy. The children and their parents were invited to the clinic. The sample selected included 112 consecutive patients with a history of CNLDO. The parents signed informed consent for the participation of their child in the study and filled in specially designed questionnaires, related to demographic characteristics and laterality of CNLDO.

The children were 1.8 to 13 years of age during the control examinations, which aimed to determine the refractive status of the child. The mean follow-up period was 5.2 years ( 0.6 to 11.6 years). Children were divided into two groups according to the laterality of the disease: group 1 included children with unilateral CNLDO and group 2, including children with bilateral obstruction. The patients were examined by the author $(\mathrm{KV})$. A control examination of the evaluated children included visual acuity (for verbal ones), evaluation of the refractive status and cycloplegic refraction in children with a low vision. The visual acuity exam was made on a light table, reflected on a mirror $2.5 \mathrm{~m}$ on the opposite. Refractive status was detected by autorefractometry with ARM ChaRops CRK
7000. We induced cycloplegia (Cyclogyl 3X every 15 minutes) if any refraction error and low visual acuity were found. Following the exam, glasses were prescribed, and the vision was tested again.

The identification of children having risk factors for amblyopia was based on referral criteria adopted by the American Association for Pediatric Ophthalmology and Strabismus (AAPOS): spherical or cylindrical anisometropia $>1.5$ diopters (D); astigmatism more than 1.5 $\mathrm{D}$ in case of with-the-rule and against-the-rule astigmatism, and it is more than $1.0 \mathrm{D}$ in case of oblique astigmatism; hypermetropia is above $3.5 \mathrm{D}$; myopia $>3.0 \mathrm{D}$, presence of manifest strabismus, and eye media opacity, and ptosis [10].

If the visual acuity of both eyes was not adjusted to 1.0 after correction with glasses in verbal children, then amblyopia was diagnosed.

The data from the examinations was statistically mapped out and analyzed using Microsoft Excel 2010 and StatGraph 2.0.0.0. The declared p-values came from the t-test, and $\mathrm{p}<0.05$ was statistically significant.

\section{Results}

Of the 224 children, only $112(50.0 \%)$ of the examined children and their parents responded. The demographic profile of the evaluated children was: $55(49.1 \%)$ males and $57(50.9 \%)$ females; $94(8483.9 \%)$ children living in urban areas and $18(1616.1 \%)$ in rural areas; $79(70.5 \%)$ children had unilateral obstruction. The right eye was affected in 37 children $(46.8 \%)$ and the left - in 42 (53.2\%). The children with bilateral CNLDO after birth were 33 (29\% 29.5\%). The average age of the children during the period of testing was 12 months (range 2-41 months).

Children were divided into two groups: Group 1, including children with unilateral CNLDO, and Group 2, including children with bilateral obstruction.

In all children examined, we found normal visual acuity in $85(75.9 \%)$ and low visual acuity in $15(13.4 \%)$. We could not examine the visual acuity in $12(10.7 \%)$ nonverbal children, but autorefractometry did not reveal refractive errors in these children. The visual acuity of the children in Group 1 and 2 is shown in Table 1.

All of the fifteen children with low visual 
acuity were prescribed appropriate optical correction after cycloplegia. Two of them (one with unilateral CNLDO and one with bilateral CNLDO) did not adjust visual acuity to 1.0 on both eyes. They had low degree amblyopia due to oblique astigmatism.

The most frequent refractive error found among the 112 studied children was hyperopia in $72(64.3 \%)$, followed by astigmatism in $17(15.2 \%)$, a combination of hyperopia and astigmatism in $10(8.9 \%)$, myopia in $10(8.9 \%)$, and a combination of myopia and astigmatism in $3(2.7 \%)$. In the two groups, the refractive state is divided as follows (Figure 1). The refractive state is divided in the two groups. In Group 1 there are $54(68.4 \%)$ children with hyperiopia, $8(10.1 \%)$ with myopia, $9(11.4 \%)$ with astigmatism, 7 $(8.8 \%)$ with combination of hyperopia and astigmatism, and $1(1.3 \%)$ with combination of myopia and astigmatism. In Group 2 children with hyperiopia are $18(54.5 \%)$, with myopia$2(6.1 \%)$, with astigmatism are $8(24.2 \%)$, with combination of hyperopia and astigmatism are $3(9.1 \%)$, and with combination of myopia and astigmatism are $2(6.1 \%)$ children (Figure 1$)$.

We detected only refraction disorders during the assessment of amblyogenic risk factors.

Table1. Visual acuity of children in Group 1 and 2 (Number, \%)

\begin{tabular}{llll}
\hline & $\begin{array}{l}\text { Group 1 } \\
\text { /Unilateral cases/ }\end{array}$ & $\begin{array}{l}\text { Group 2 } \\
\text { /Bilateral cases/ }\end{array}$ & Total \\
\hline Normal visual acuity (vis=1.0) & $61(77.2)$ & $24(72.7)$ & $85(75.9)$ \\
& $9(11.4)$ & $6(18.2)$ & $15(13.4)$ \\
Low visual acuity $($ vis $<\mathbf{0 . 8 - 1 . 0})$ & $9(11.4)$ & $3(9.1)$ & $12(10.7)$ \\
$\begin{array}{l}\text { No visual acuity } \\
\text { in nonverbal children }\end{array}$ & $79(70.5)$ & $33(29.5)$ & $112(100.0)$ \\
Total & &
\end{tabular}

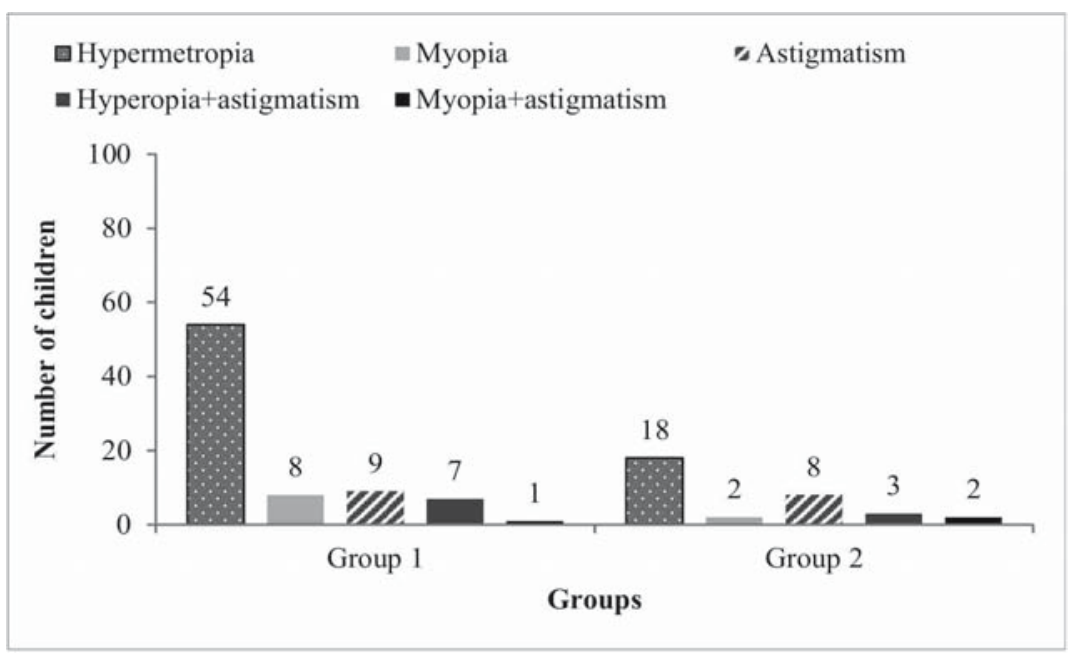

Figure 1. Frequency of different types of refractive state in Group 1 and Group 2

There was no strabismus, media opacity or ptosis in the children evaluated. The risk factors for development of amblyopia in our study included: astigmatism $>1.0 \mathrm{D}$ in the oblique axis in 4 children - three children $(3.8 \%)$ in Group 1 , and one child (3.0\%) in Group 2; cylindrical anisometropia $>1.5 \mathrm{D}$ in 3 children - two children (2.5\%) in Group 1 and one child (3.0\%) in Group 2); myopia > 3.0 D in 2 children - one child (1.3\%) in Group 1 and one child (3.0\%) in
Group 2.

Comparing eyes affected by CNLDO and those non-affected in Group 1, we did not find significant differences in the values for diopters sphere $(\mathrm{DSPH})(\mathrm{p}=0.959)$ and the diopters cylinder (DCYL) between both eyes $(p=0.212)$ - Table 2.

Comparing the inter-ocular difference of spherical and cylindrical refractive error in 
Table 2. Difference between affected and non-affected fellow eyes in mean values of refractive indices in Group 1

\begin{tabular}{|c|c|c|c|c|c|}
\hline & Affected eye & & Non-affected fellow eyes & & p-value \\
\hline & Mean value & SD & Mean value & SD & \\
\hline DSPH & $\begin{array}{l}-0.02 \\
( \pm 0.26) \\
\min -3.5, \max +1.5\end{array}$ & 1.01 & $\begin{array}{l}-0.03 \\
( \pm 0.25) \\
\min -2.5, \max +3.0\end{array}$ & 0.99 & 0.950 \\
\hline DCYL & $\begin{array}{l}0.13 \\
( \pm 0.18) \\
\min -1.25, \max +3.0\end{array}$ & 0.69 & $\begin{array}{l}0.26 \\
( \pm 0.15) \\
\min -1.0, \max +2.25\end{array}$ & 0.61 & 0.212 \\
\hline
\end{tabular}

Group 2 patients in with bilateral CNLDO, we established a statistically insignificant difference between DSPH of the right eye and left eye $(p=0.925)$, and DCYL of the right eye and left

eye $(p=0.740)$ - Table 3 .

\section{Discussion}

Table 3. Difference between right and left eye in mean values of refractive indices in Group 2

\begin{tabular}{|c|c|c|c|c|c|}
\hline & Right eye & & Left eye & & p-value \\
\hline & Mean value & $\mathrm{SD}$ & Mean value & SD & \\
\hline DSPH & $\begin{array}{l}-0.46 \\
( \pm 0.65) \\
\min -4.0, \max +1.25\end{array}$ & 1.46 & $\begin{array}{l}-0.43 \\
( \pm 0.48) \\
\min -3.25, \max +1.0\end{array}$ & 1.11 & 0.925 \\
\hline DCYL & $\begin{array}{l}0.15 \\
( \pm 0.25) \\
\min -2.0, \max +1.0\end{array}$ & 0.67 & $\begin{array}{l}0.09 \\
( \pm 0.29) \\
\min -2.75, \max +1.0\end{array}$ & 0.79 & 0.740 \\
\hline
\end{tabular}

CNLDO affects one eye (left or right) in about two-thirds of such patients, and both eyes are affected in the remainder [7, 11-13]. In our study, the laterality of CNLDO was similar: $29.5 \%$ of individuals with bilateral CNLDO, $46.8 \%$ with affected right eyes and 53.2\% with affected left eyes. The most frequent refraction anomaly $(64.3 \%)$ in the studied children was hyperopia, which corresponds to the literature data $[7,13]$.

Some authors have shown that the risk of development of amblyopia in children with CNLDO is high $[4,5,14]$. Of the 433 children investigated by Eshraghi et al. (2014), $9.5 \%$ were found to have amblyopia risk factors [4]. In the study of Matta et al. (2010), who examined 402 children with CNLDO, the preponderance of amblyopia risk factors was $22.0 \%$ [5]. Kim et al. reported a higher percentage of presence of this risk factor $-35.0 \%$, but their sample size was too small-only 26 children [14].

We found that $8.0 \%$ of the children we studied were with amblyopia risk factors: $3.6 \%$ with astigmatism $>1.0 \mathrm{D}$ in the oblique axis, $2.7 \%$ with anisometropia, and $1.8 \%$ with moderate myopia. The above-cited studies used the same referral criteria of AAPOS for detecting amblyopia risk factors.

According to the literature, the presence of anisometropia seems to be more common in children with CNLDO, as compared with the general population [7]. The prevalence of anisometropia ranges between 1.4 and $3.4 \%$ in natural populations [15-19]. The rate of anisometropia in patients with CNLDO, reported in publications varies from $5.5 \%$ to $19.0 \%$ [4, $5,7,9]$. In our research, anisometropia was found in $2.682 .7 \%$ of the examined children. This percentage is considerably lower than the one reported by other authors. In our patients, anisometropia was associated with astigmatism, whereas other studies have reported an association with hyperopia [6, 7]. Laterality is an essential factor for the development of differences between refraction in the two eyes. Many authors have emphasised on the association of unilateral CNLDO and anisometropia $[6,7,20]$. Patients with bilateral CNLDO are at a much lower risk of developing significant anisometropia, as well as anisometropic amblyopia [9]. In our study, unilateral and bilateral CNLDO had the same effect on the refraction of both eyes. There was no significant differences $(p>0.05)$ between the 
spherical and cylindrical refractive status of the two eyes.

Many authors have reported that amblyopia is more common in children with CNLDO and that anisometropia is the contributing factor $[5,7,20$, 21]. According to different studies, the incidence of amblyopia varies between $5.2 \%$ and $10.0 \%$ $[7,8]$. In natural populations, anisometropic amblyopia varies between 0.7 and $1.3 \%$ [15-19]. We found low-grade amblyopia in two of the examined children $(1.8 \%)$ and the reason for it in both cases was oblique astigmatism $(>1.0 \mathrm{D})$.

However, no cause-effect connection between CNLDO and anisometropia/amblyopia has been established by the cited studies. The mechanism by which patients with CNLDO might develop anisometropia and/or amblyopia is still unclear. Nevertheless, it is suggested that increased tear meniscus, disturbances in the tear film, and mucopurulent discharge cause disturbance in image formation during the critical period of visual development $[5,8]$. If this is a causal relationship, it might significantly modify the way of treatment, supporting earlier probing rather than a conservative approach [22]. In our study, the average age of the children during the probing performed was 12 months, and no statistically significant correlation between CNLDO and refraction anomalies was established. Therefore, later probing in children with CNLDO would not be necessarily associated with the development of anisometropia or amblyopia.

According to some authors, the presence of obstruction of the NLD does not affect the development of the visual system in children. Ellis et al. (1998) were the first to consider a correlation between CNLDO and potential vision problems. They found no evidence that disruption of the tear film associated with CNLDO interferes with emmetropization. They found no significant differences in amblyopia or hypermetropia rates in the tearing and nontearing eye in their CNLDO group. Furthermore, there were no significant differences in the overall frequency of amblyopia or hypermetropia between children in the CNLDO and the controls [23]. These results are the same as in our study.

There are a few limitations to this work. First, the study was based only on the patients who accepted to participate $(50.0 \%$ of all children we had registered with CNLDO). Second, we could not select a control group of healthy children.
Third, we applied cycloplegia only on children with low visual acuity. Besides, we had no data about the refractive status of the children before the probing for CNLDO.

\section{Conclusions}

We found no information for the negative outcome of unilateral or bilateral CNLDO on visual improvement, yet amblyogenic risk factors were higher in the patients we studied. Thus, a more comprehensive assessment should be carried out to identify and follow-up patients with these risk factors. Careful eye examination, including objective determination of the refraction with cycloplegia, could contribute to early prevention of amblyopia.

\section{Acknowledgements}

This work was supported by Medical University - Pleven, Bulgaria. The article was a part of Project No BG05M2OP001-2.009-0031-C01.

\section{References}

1. Chernodrinska V. [The most common eye diseases in children. In: The most common congenital ocular defects and diseases in children]. 1st ed. Sofia: Bulvest 2000; 2016. Bulgarian.

2. MacEwen CJ, Young JD. Epiphora during the first year of life. Eye. 1991;5(P1 5):596-600.

3. Yuen SJ, Oley C, Sullivan TJ. Lacrimal outflow dysgenesis. Ophthalmology. 2004;111(9):178290.

4. Eshraghi B, Akbari MR, Fard MA, Shahsanaei A, Assari R, Mirmohammadsadeghi A. The prevalence of amblyogenic factors in children with persistent congenital nasolacrimal duct obstruction. Graefes Arch Clin Exp Ophthalmol. 2014;252(11):1847-52.

5. Matta NS, Singman EL, Silbert DI. Prevalence of amblyopia risk factors in congenital nasolacrimal duct obstruction. J AAPOS. 2010;14(5):386-8.

6. Bagheri A, Safapoor S, Yazdani S, Yaseri M. Refractive state in children with unilateral congenital nasolacrimal duct obstruction. J Ophthalmic Vis Res. 2012;7(4):310-5.

7. Piotrowski JT, Diehl NN, Mohney BG. Neonatal dacryostenosis as a risk factor for anisometropia. Arch Ophthalmol. 2010;128(9):1166-9. 
8. Lacey BA, McGinnity GF, Johnston PB, Archer DB. Congenital epiphora as a potential cause of amblyopia. Vision Res. 1995; 35 Suppl 1:S130.

9. Siddiqui SN, Hannan A, Mansoor H, Asif M, Wakeel U. Anisometropia and refractive status in children with bilateral congenital nasolacrimal duct obstruction. Journal of the $\mathrm{J}$ Coll Physicians Surg Pak. 2018;28(3):210-3.

10. Donahue SP, Arnold RW, Ruben JB, AAPOS Vision Screening Committee. Preschool vision screening: what should we be detecting and how should we report it? Uniform guidelines for reporting results of preschool vision screening studies. J AAPOS. 2003;7(5):314-6.

11. Thongthong K, Singha P, Liabsuetrakul $T$. Success of probing for congenital nasolacrimal duct obstruction in children under 10 years of age. J Med Assoc Thai. 2009;92(12):1646-50.

12. Castelo Branco Neto E, Castelo Branco B, Cardoso CC, Garcia de Carvalho B, Mota E, Castelo Branco A. Management of congenital nasolacrimal duct obstruction. Arg Bras Oftalmol. 2009;72(1):75-8.

13. Ozgur OR, Sayman IB, Oral Y, Akmaz B. Prevalence of amblyopia in children undergoing nasolacrimal duct irrigation and probing. Indian J Ophthalmol. 2013;61(12):698-700.

14. Kim JW, Lee H, Chang M, Park M, Lee TS, Baek S. Amblyopia risk factors in infants with congenital nasolacrimal duct obstruction. J Craniofac Surg. 2013;24(4):1123-5.

15. Flom MC, Bedell HE. Identifying amblyopia using associated conditions, acuity, and nonacuity features. Am J Optom Physiol Opt. 1985;62(3):153-60.

16. Drover JR, Kean PG, Courage ML, Adams
RJ. Prevalence of amblyopia and other vision disorders in young Newfoundland and Labrador children. Can J Opthalmol. 2008;43(1):89-94.

17. Payman J, Akbar F, Hassan H, Masud Y, Alireza J. Refractive errors and amblyopia in children entering school: Shahrood, Iran. Optom Vis Sci. 2009;86(4):364-9.

18. William C, Harrad RA, Harvey I, Sparrow JM, ALSPAC Study Team. Screening for amblyopia in preschool children: Results of a population based, randomised controlled trial. Ophthalmic Epidemiol. 2001;8(5):279-95.

19. Pediatric Eye Disease Investigator group. The clinical profile of moderate amblyopia in children younger than 7 years. Arch Opthalmol. 2002;120(3):281-7.

20. Simon JW, Ngo Y, Ahn E, Khachikian S. Anisometropic amblyopia and nasolacrimal duct obstruction. J Pediatr Ophthalmol Strabismus. 2009;46(3):182-3.

21. Matta NS, Silbert DI. High prevalence of amblyopia risk factors in preverbal children with nasolacrimal duct obstruction. J AAPOS. 2011;15(4):350-2.

22. Matta NS, Silbert DI, Singman EL. Does nasolacrimal duct obstruction cause amblyopia? Expert Rev Ophthalmol. 2012;7(2):103-4.

23. Ellis JD, MacEwen CJ, Young JD . Can congenital nasolacrimal-duct obstruction interfere with visual development?A cohort case control study. J Pediatr Ophthalmol Strabismus. 1998;35(2):81-5. 